Review Article

\title{
Acupuncture for Spinal Cord Injury and Its Complications: A Systematic Review and Meta-Analysis of Randomized Controlled Trials
}

\author{
In Heo, ${ }^{1}$ Byung-Cheul Shin, ${ }^{2,3}$ Young-Dae Kim, ${ }^{1}$ Eui-Hyoung Hwang, ${ }^{2,3}$ \\ Chang Woo Han, ${ }^{1,3}$ and Kwang-Ho Heo ${ }^{3}$ \\ ${ }^{1}$ School of Korean Medicine, Pusan National University, Yangsan 626-870, Republic of Korea \\ ${ }^{2}$ Department of Rehabilitation Medicine, School of Korean Medicine, Pusan National University, \\ Yangsan 626-870, Republic of Korea \\ ${ }^{3}$ Korean Medicine Hospital, Pusan National University, Yangsan 626-870, Republic of Korea \\ Correspondence should be addressed to Byung-Cheul Shin; drshinbc@gmail.com
}

Received 21 October 2012; Accepted 8 December 2012

Academic Editor: Gerhard Litscher

Copyright (C) 2013 In Heo et al. This is an open access article distributed under the Creative Commons Attribution License, which permits unrestricted use, distribution, and reproduction in any medium, provided the original work is properly cited.

\begin{abstract}
To evaluate the evidence supporting the effectiveness of acupuncture treatment for SCI and its complications, we conducted search across 19 electronic databases to find all of the randomized controlled trials (RCTs) that used acupuncture as a treatment for SCI and its complications. The methodological quality of each RCT was assessed using the Cochrane risk of bias tool and the PEDro scale. Sixteen RCTs, including 2 high-quality RCTs, met our inclusion criteria ( 8 for functional recovery from SCI, 6 for bladder dysfunction, and 2 for pain control). The meta-analysis showed positive results for the use of acupuncture combined with conventional treatments for the functional recovery in terms of motor ASIA scores and total FIM scores when compared to conventional treatments alone. Positive results were also obtained for the treatment of bladder dysfunction, in terms of the total efficacy rate, when comparing acupuncture to conventional treatments. However, 2 RCTs for pain control reported conflicting results. Our systematic review found encouraging albeit limited evidence for functional recovery, bladder dysfunction, and pain in SCI. However, to obtain stronger evidence without the drawbacks of trial design and the quality of studies, we recommend sham-controlled RCTs or comparative effectiveness research for each condition to test the effectiveness of acupuncture.
\end{abstract}

\section{Introduction}

Spinal cord injury (SCI) affects approximately 900 to 1,000 individuals per million in the general population, and it is estimated that there are 12,000 new cases of SCI every year in the United States [1]. Nearly $80 \%$ of the individuals who experience SCI are male, and, since 2000, the average age at injury has increased to 39.5 years, with $11.5 \%$ of those injured greater than 60 years of age [2]. These statistics indicate that SCI may be a significant social and economic burden for patients and their families.

SCI causes lesions, damaged neurological structures, and secondary pathophysiological changes in spared tissue [19]. Thus, complete or partial loss of sensory and motor function is the most significant result of injury, and most patients experience secondary complications, such as bladder and bowel dysfunction, chronic pain, infertility, and autonomic dysfunction [19].

There are many treatments for functional recovery from injury and the related complications that result from SCI; these include surgery [20], prescription drugs [21], behavioral therapy [22], physical therapy [23], and supportive treatment [24]. There are also a variety of treatments used for the secondary complications of SCI, such as intermittent catheterization for bladder dysfunction, analgesics for pain, and others [25]. These treatments tend to be administered over long periods of time, and because of the potential complications of treatment, there has been an increased interest in alternative medical treatments, including acupuncture and other related therapies (moxibustion and acupressure) [26]. Acupuncture 
based on Traditional Chinese Medicine has been commonly used for pain or neurological problems in Chinese cultures [27]. Additionally, many studies [28, 29] have analyzed the use of acupuncture for these types of problems; these studies report a variety of outcomes regarding level of function, pain, and quality of life.

Three recently published reviews have examined the results of studies that support the use of treatments such as acupuncture for individuals with SCI [26, 30, 31]. One review [30] that was limited to a Chinese literature suggested that acupuncture treatments have a positive effect, while a second [26] did not systematically evaluate the available evidence and even failed to include several important trials $[3,5-13,15,16,18]$. The third review was restricted to the pain related to SCI [31]. Therefore, the purpose of this systematic review was to provide a qualitative analysis of all of the randomized controlled trials (RCTs) to date that were designed to determine the effectiveness of acupuncture for patients with SCI and its complications along PRISMA guidelines [32].

\section{Methods}

2.1. Data Sources. This systematic review included studies published in electronic databases over the time period ranging from their inception to December 2011. Relevant publications were found in the following databases: the Cochrane Central Register of Controlled Trials (CENTRAL), the Cochrane Database of Systematic Review (CDSR), PubMed, MEDLINE, EMBASE, CINAHL, nine Korean databases (Korean Studies Information, DBPIA, Korea Institute of Science Technology Information, Korean National Assembly Library, RISS4U, KoreaMed, Korean medical database, Korean Traditional Knowledge Portal, and Oasis), Chinese database (China National Knowledge Infrastructure), and two Japanese databases (J-STAGE and JAMAS). In addition, we searched databases that contained registered trials, such as ClinicalTrials.gov (http://www.clinicaltrials.gov).

The search keywords used were (acupuncture OR acup* OR "electroacupuncture" OR "auricular acupuncture" OR "scalp acupuncture") AND ("spinal cord injury" OR "spinal injury" OR "spinal cord trauma" OR "spinal cord lesion" OR "spinal cord damage" OR "spinal cord fracture" OR "spinal cord contusion") in each base language. This search strategy was adjusted for each database. In addition, the bibliographies of relevant systematic reviews and clinical guidelines were manually searched. We also searched the gray literature that included dissertations, letters, government documents, research reports, conference proceedings, and abstracts when available. The reference section for each study was also searched.

\subsection{Study Selection}

2.2.1. Types of Studies. We evaluated RCTs that studied the clinical effects of acupuncture as a treatment for SCI or its direct complications. Dissertations and abstracts examining these topics were also included. The review included prospective, parallel RCTs, or cross-over RCTs that assessed the efficacy of acupuncture regardless of blinding, language, or the type of report. The title and abstract of each identified article were read by a single primary researcher $(\mathrm{IH})$ who completed the screening process. If articles were not written in English, they were translated into English prior to screening. The articles that would then be potentially included in our analysis were carefully checked by 2 independent reviewers (IH, YDK).

2.2.2. Types of Participants. The clinical trials included in our review examined patients with SCI or complications secondary to SCI. There were no restrictions related to the amount of time after injury, the type of injury, the site of injury, or participant's age.

2.2.3. Types of Interventions. Our study considered the effects of needle acupuncture. We included needle acupuncture with or without electrical stimulation or heating by moxa, auricular acupuncture, and scalp acupuncture. We excluded injection acupuncture, nonneedling acupuncture (e.g., laser acupuncture), acupuncture-like intervention (e.g., acupressure, moxibustion), and mixed treatments. Control conditions in the reviewed studies included sham acupuncture, no treatment, and conventional treatment for SCI (e.g., medication, rehabilitation). Studies using cointerventions were included only if cointerventions were given to both treatment groups.

2.2.4. Outcome Measures. The outcome measures we considered were neurologic status or score (i.e., the American Spinal Injury Association neurologic and functional score; the ASIA score), functional ability outcomes (i.e., the FuglMeyer score), activities of daily living (i.e., the Functional Independence Measure; FIM score), scores related to the efficacy rate (i.e., the rate of participants who demonstrated efficacy), and outcome measures related to the complications of SCI (i.e., the bladder function scale for bladder dysfunction, pain scores for the level of pain and range of motion; ROM). We also considered measures of general safety reported for acupuncture as a treatment.

\subsection{Data Extraction and Quality Assessment. Two reviewers} (IH, YDK) reviewed each article independently and were blinded to the findings of the other reviewer. The reviewers collected data relating to the methodology, outcome measures, results, and final conclusions of each study. The reviewers also quantitatively evaluated each study's methodological quality through the use of the Cochrane risk of bias [33] and the Physiotherapy Evidence Database (PEDro) scale [34], which was developed to assess the methodological quality of RCTs specifically pertaining to physical therapy interventions [35]. The PEDro scale allows researchers to assess the quality of studies based on a cutoff score: studies that score fewer than six points are considered to be "low quality", while studies with a score equal to or greater than six points are considered to be "high quality" [36]. Any discrepancies 
between reviewers were resolved through discussion until a consensus was reached.

2.4. Data Synthesis. To summarize the effects of acupuncture on each outcome measure, we used Cochrane Collaboration software (Review Manager (RevMan) Version 5.1 for Windows. Copenhagen: The Nordic Cochrane Centre). We extrapolated the risk estimate (relative risk; RR) and the 95\% confidence interval (CI) for dichotomous data. The standard mean difference (SMD) and the 95\% CI were calculated for continuous data. The variance of the amount of change was imputed using a correlation factor of 0.4 , which is the value suggested by the Cochrane Collaboration [33]. We pooled data across studies using random effect models if excessive statistical heterogeneity did not exist. The chi-squared test and the Higgins $I^{2}$ test were used to assess the heterogeneity of the data [33]. We planned to use a formal funnel plot to assess publication bias if more than 10 trials were included; however, we were unable to generate a funnel plot due to the small number of trials included in our meta-analysis.

\section{Results}

3.1. Study Description. We considered 960 potentially relevant articles. After screening the abstracts and titles, we excluded 557 studies (Figure 1). Forty-seven articles were fully evaluated. Thirty-one additional articles were subsequently excluded; 5 studies were not controlled and 13 studies were not randomized trials. Thirteen RCTs were also excluded: 3 of them compared 2 types of acupuncture [3739], 2 analyzed injection acupuncture [40, 41], 3 included mixed treatments [42-44], 3 included patients other than those with SCI [45-47], 1 provided an insufficient report [48], and 1 used acupressure as opposed to acupuncture [49]. Consequently, 16 RCTs met our inclusion criteria [318]. Eight trials studied functional recovery in SCI (5 in traumatic SCI [4-6, 8, 10], 1 in nontraumatic SCI [7], and 2 were not reported $[3,9]), 6$ trials studied bladder dysfunction secondary to SCI (1 in traumatic SCI [13], 1 in mixed SCI [15], and 4 were not reported $[11,12,14,16])$, and 2 trials studied the use of acupuncture for pain control in SCI (2 trials did not report SCI type) $[17,18]$. The main data are summarized in Table 1. There were 12 Chinese studies [3, 5-13, 15, 16], 2 Taiwanese studies $[4,14]$ and 2 American studies $[17,18]$. Fifteen studies used 2-parallel-arm group designs [3-13, 1518 ] and 1 used a 4-parallel-arm group design [14].

3.2. Study Quality. The mean PEDro score was 4.5; scores ranged from 4 to 8 points (Table 2). Only 2 of 16 RCTs met the PEDro criteria for high quality $[9,17]$. The results of the Cochrane risk of bias analysis varied widely (Table 2). Two studies reported appropriate sequence generation $[17,18]$, in which the researchers used a coin toss method [18] and stratified block randomization [17]. Two of the trials reported inappropriate randomization procedures $[8,16]$, in which the researchers used hospital admission numbers for randomization. One trial blinded the participants and the assessors [17], and three blinded the assessors only $[4,9,18]$. Only one trial mentioned that the outcomes were analyzed with an intention-to-treat analysis [17]. Incomplete outcome data were addressed adequately in three studies $[14,17,18]$. Only one trial implemented allocation concealment; however, a detailed description of the method was not reported [9].

3.3. Descriptions of Acupuncture Treatment. The majority of the included RCTs stated that the rationale for acupuncture point selection was drawn from Traditional Chinese Medicine theory (Table 3). Fourteen studies used electrical stimulation with acupuncture [3-16] and 2 studies used needling acupuncture alone $[17,18]$. Ten trials used fixed acupuncture points $[4,5,7,9-14,16], 5$ trials used fixed plus individualized acupuncture points $[6,8,15,17,18]$, and 1 trial chose individualized by symptoms acupuncture points [3]. A total of 48 acupuncture points were included in functional recovery (meridian points: 36 , extra point: 1, ear acupuncture points: 8, scalp acupuncture points: 2, Ashi point: 1), and $62.5 \%$ were located in the upper and lower extremities, $37.5 \%$ in head and back. A total of 18 acupuncture points were included in bladder dysfunction (meridian points: 17, extra point: 1) and $77.8 \%$ were located in the lower back. A total of 30 acupuncture points were included in pain control (meridian points: 29, Ashi point: 1 ) and 93.3\% were located in shoulder area for the shoulder pain control of SCI.

\subsection{Outcomes}

\subsubsection{Functional Recovery}

Acupuncture versus Conventional Treatment (1 RCT). One RCT evaluated the effect of electrical acupuncture compared to rehabilitation [3]. A significant difference was found between the two groups regarding the total efficacy rate $(P=$ 0.05 , Table 1).

Acupuncture Plus Conventional Treatment versus Conventional Treatment (7 RCTs). Five RCTs evaluated the effects of electrical acupuncture plus rehabilitation and compared the results to those of rehabilitation alone $[4-7,9]$. Three RCTs described improved outcomes with electrical acupuncture plus rehabilitation over rehabilitation alone $[4,7,9]$. However, 2 RCTs did not show any significant differences between treatment groups (Table 1) $[5,6]$.

One RCT compared the effects of electrical acupuncture, rehabilitation, and oral neurotropic drugs to those of rehabilitation and oral neurotropic drugs [8]. This study showed a positive effect in terms of rehabilitation effectiveness only $(P=0.05$, Table 1$)$.

One RCT evaluated the effects of electrical acupuncture plus intravenous drugs compared to intravenous drugs alone [10]. This RCT showed that treatment consisting of electrical acupuncture plus intravenous drugs was more effective than intravenous drugs alone $(P=0.003)$.

The meta-analysis of acupuncture plus conventional treatment versus conventional treatment alone showed that treatment including acupuncture led to significantly more improved motor ASIA scores (2 studies [4, 7]: $n=156$, SMD $=0.78,95 \%$ CI of 0.36 to $1.20, P=0.0002$, heterogeneity: 


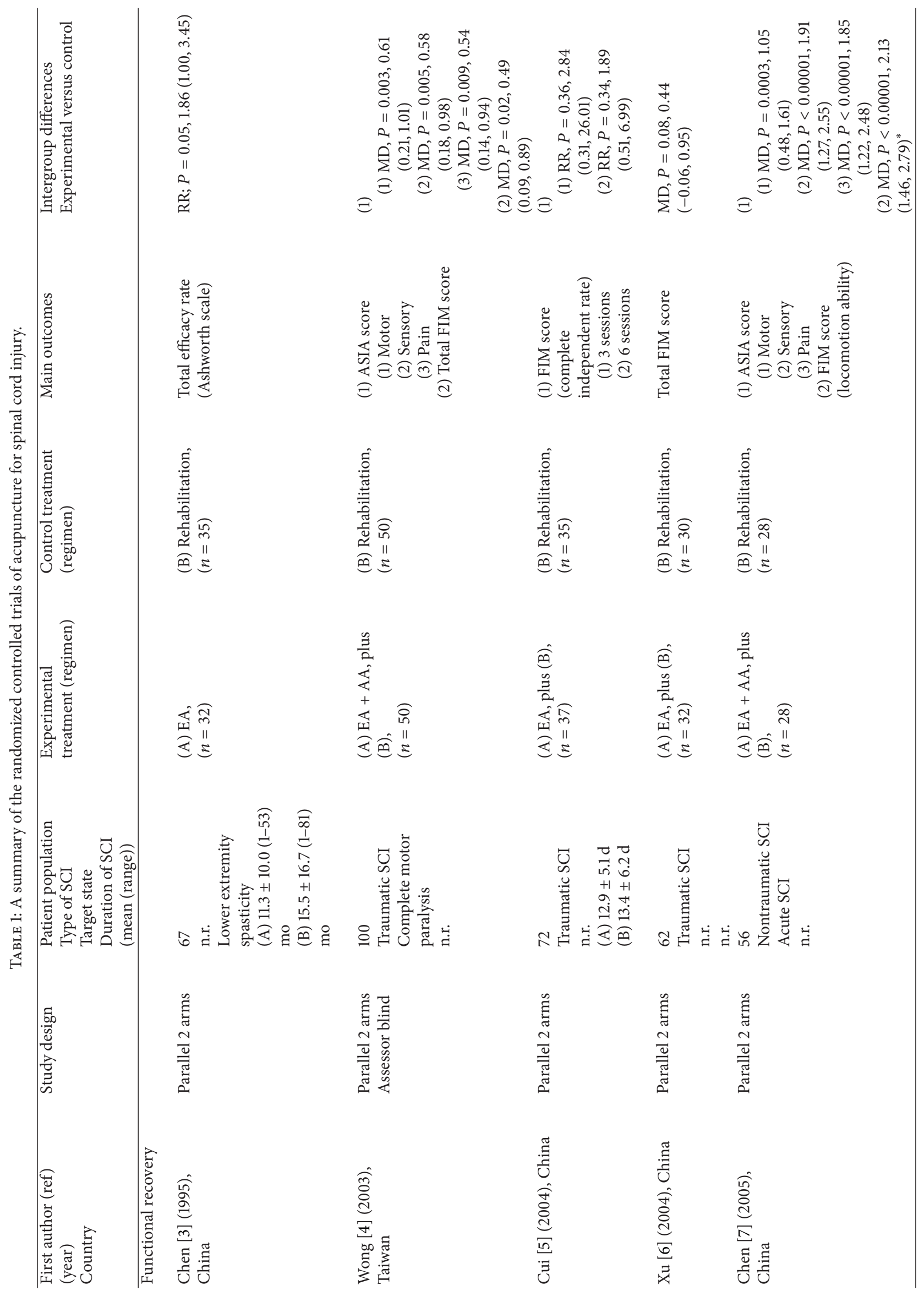




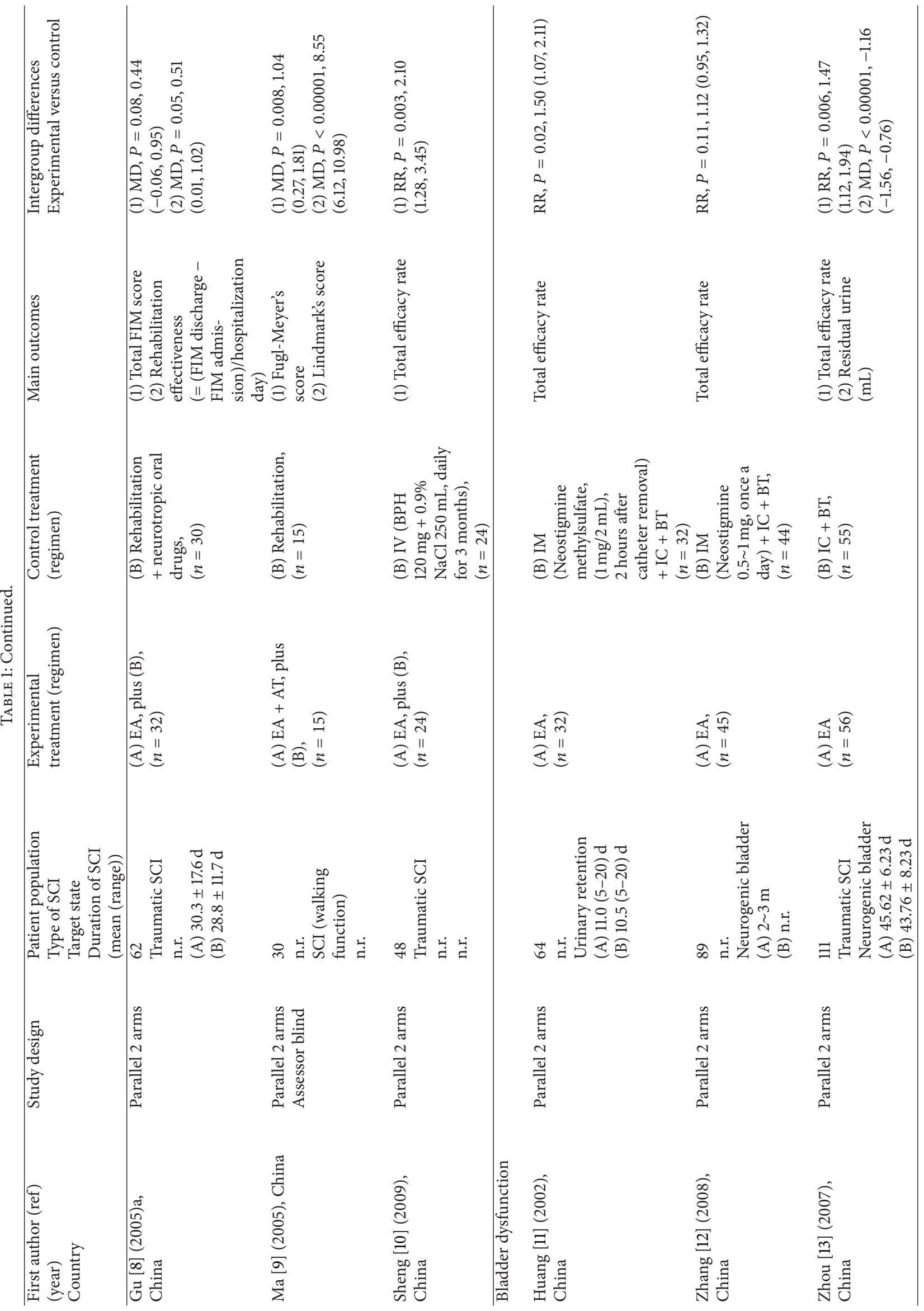




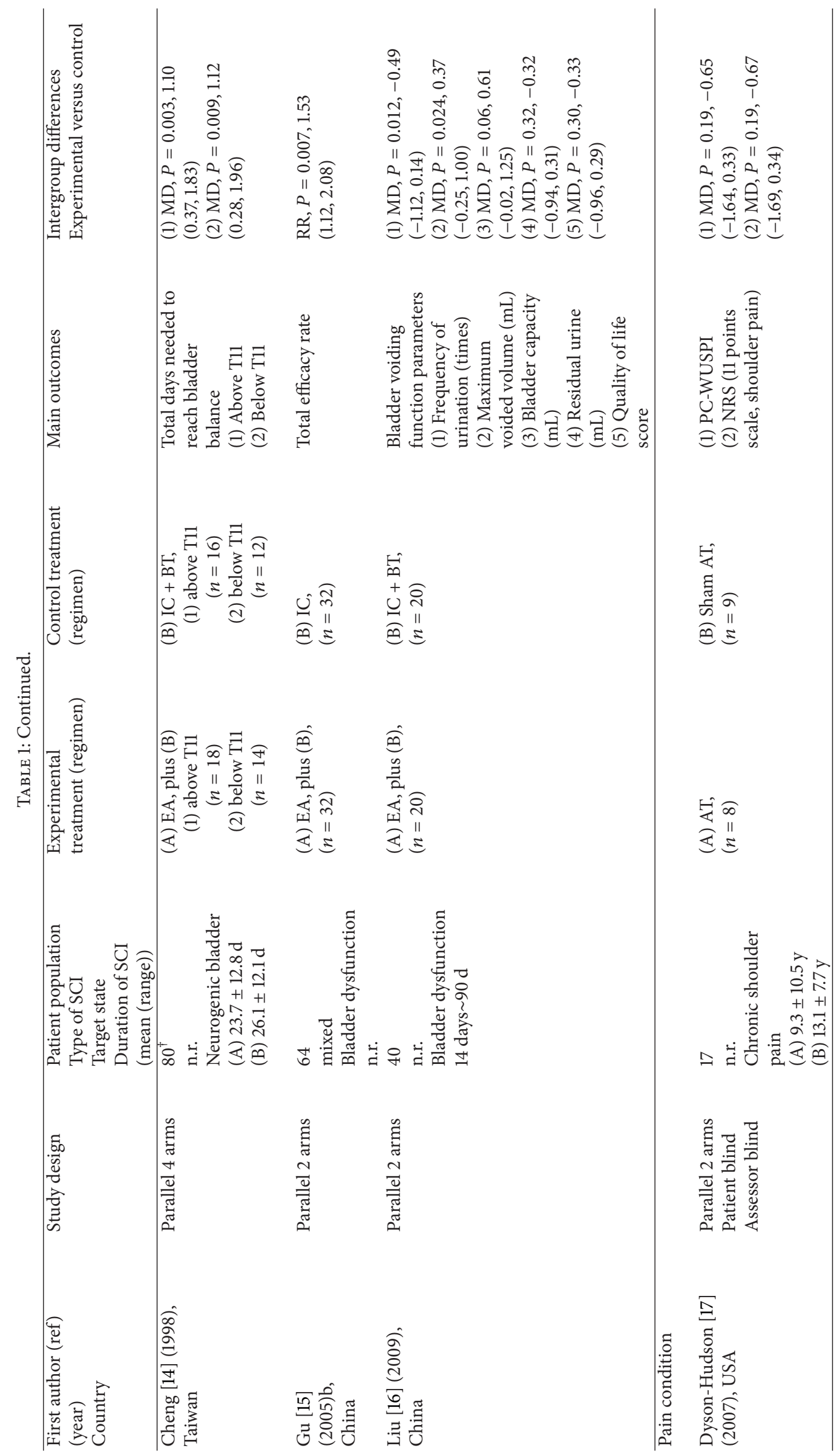




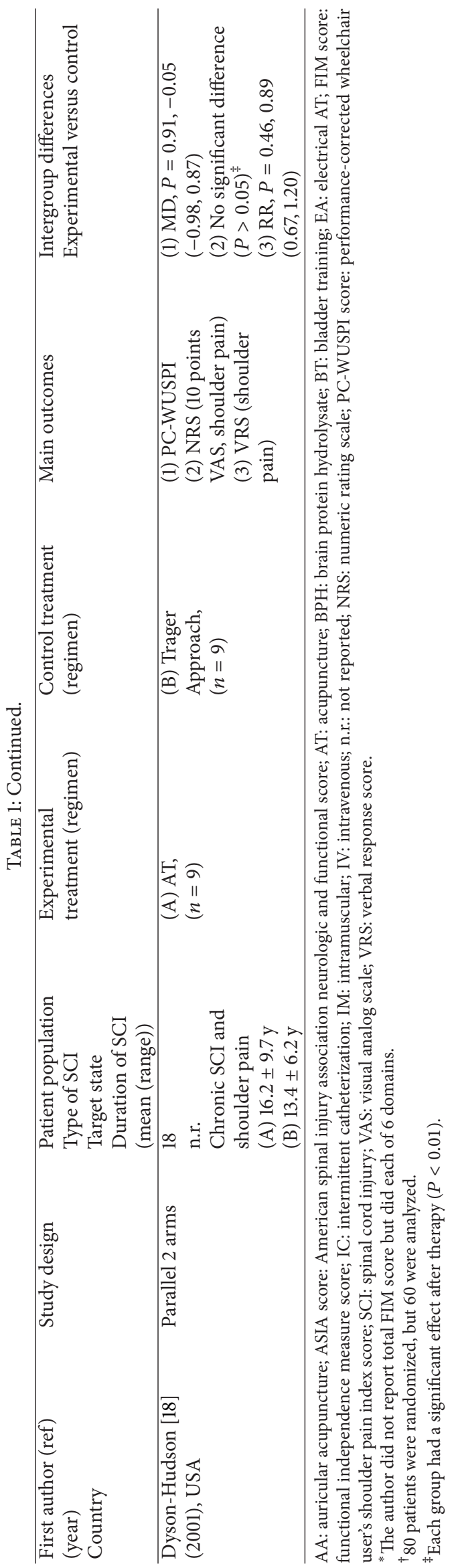




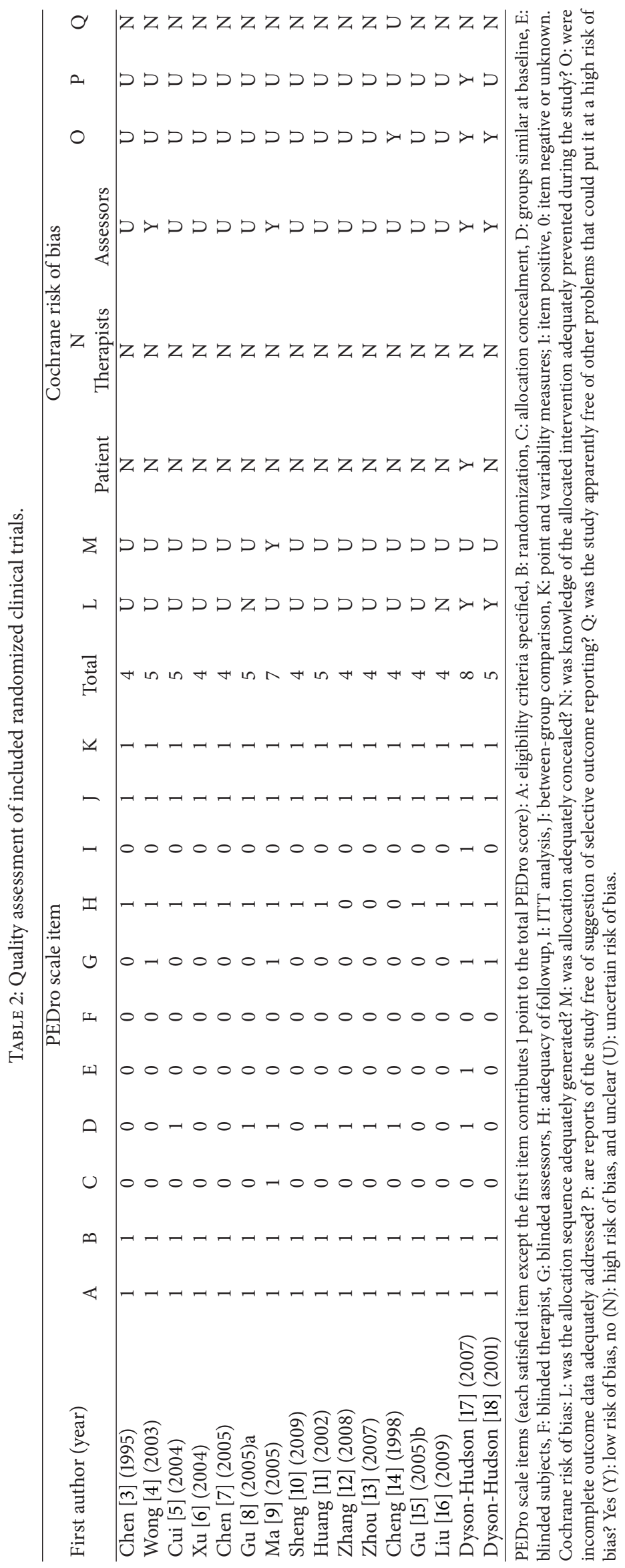




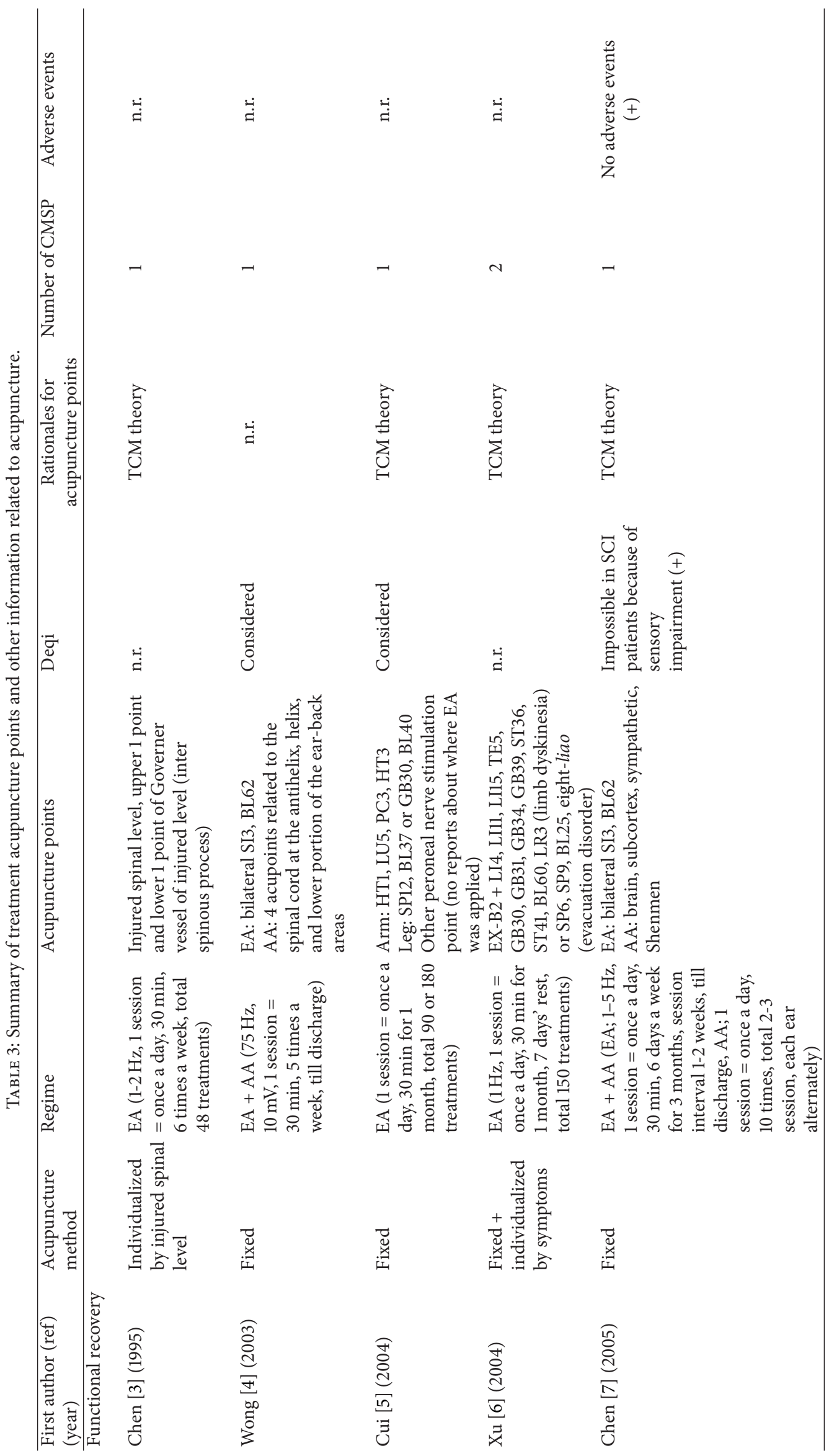



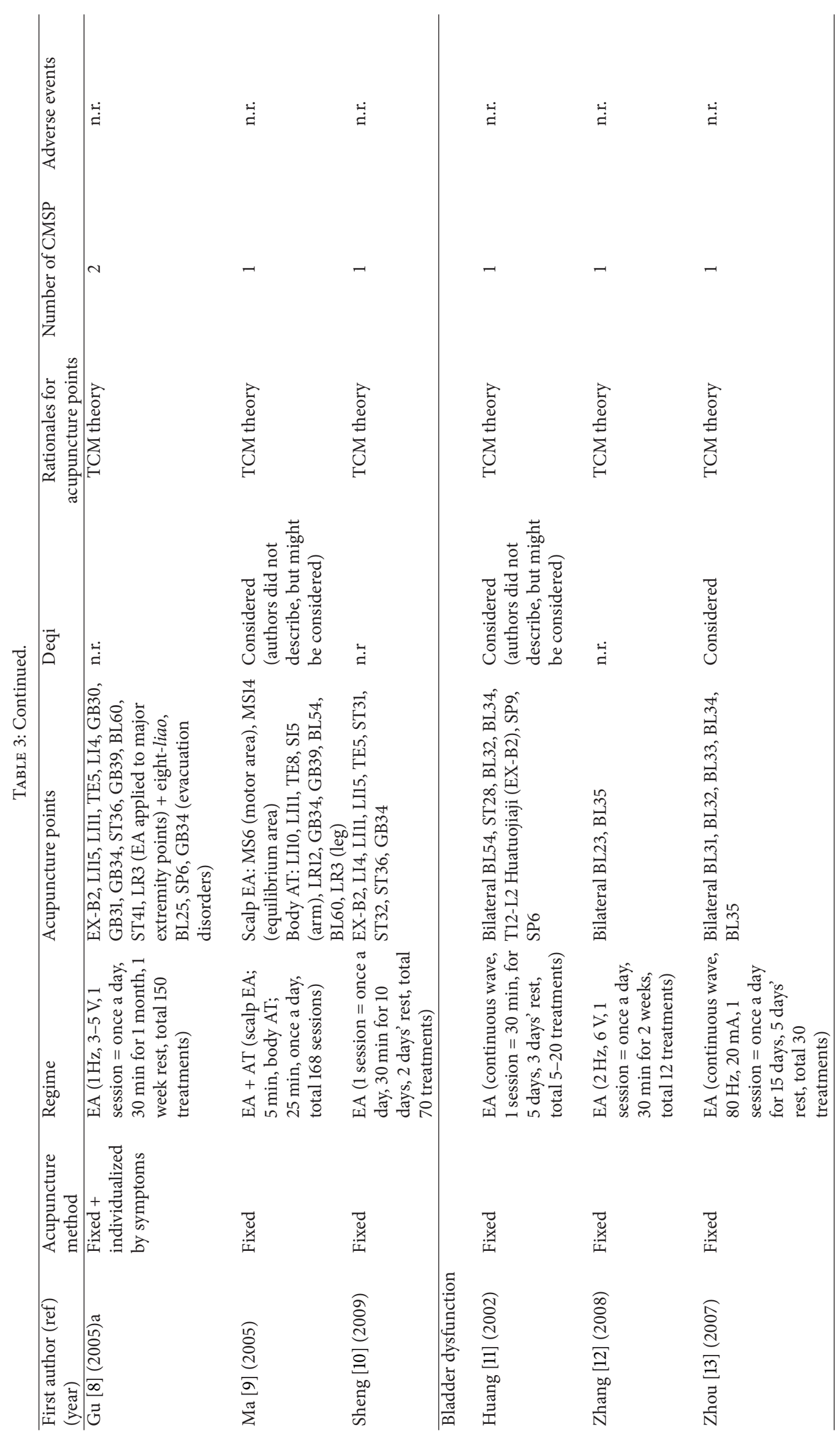


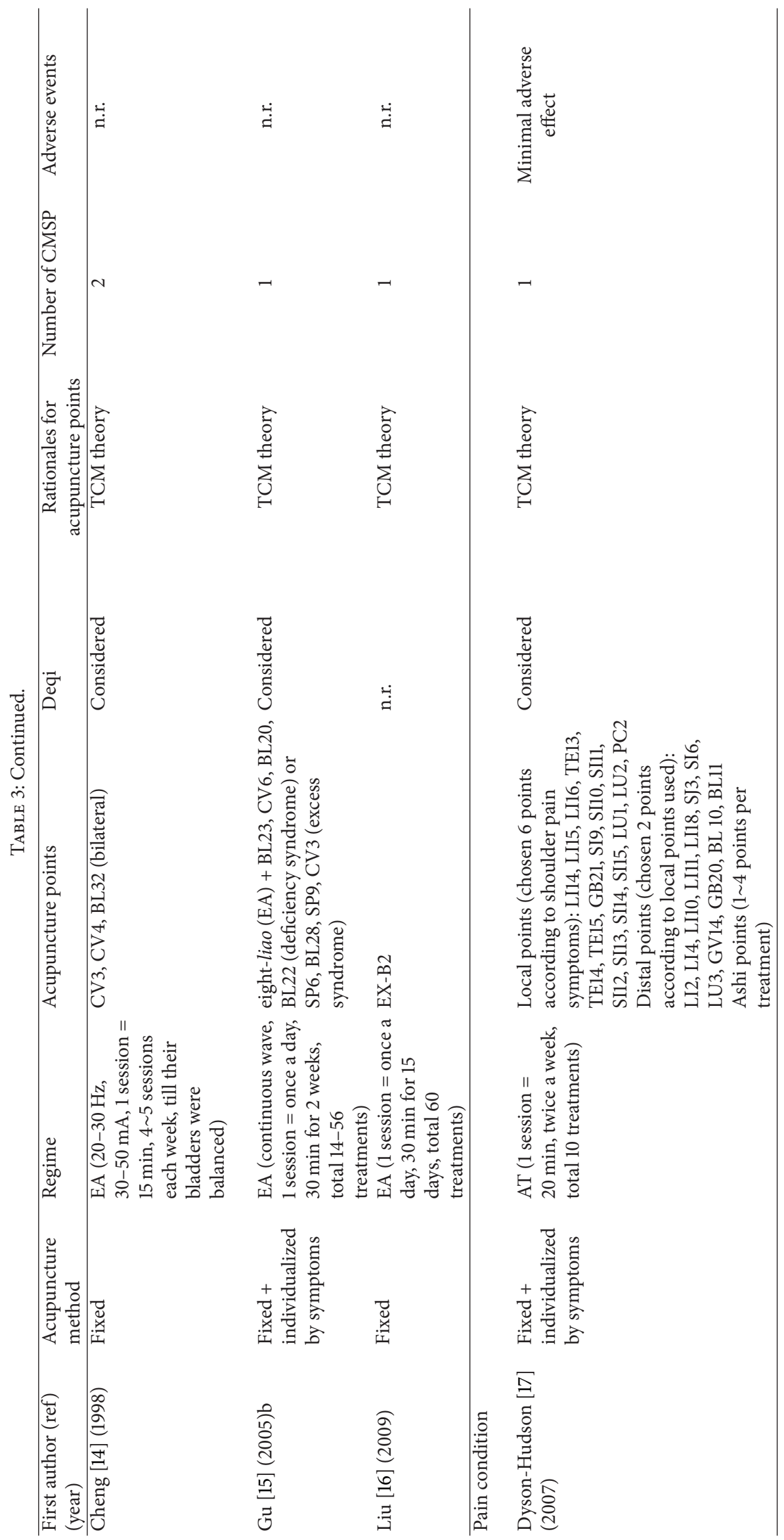




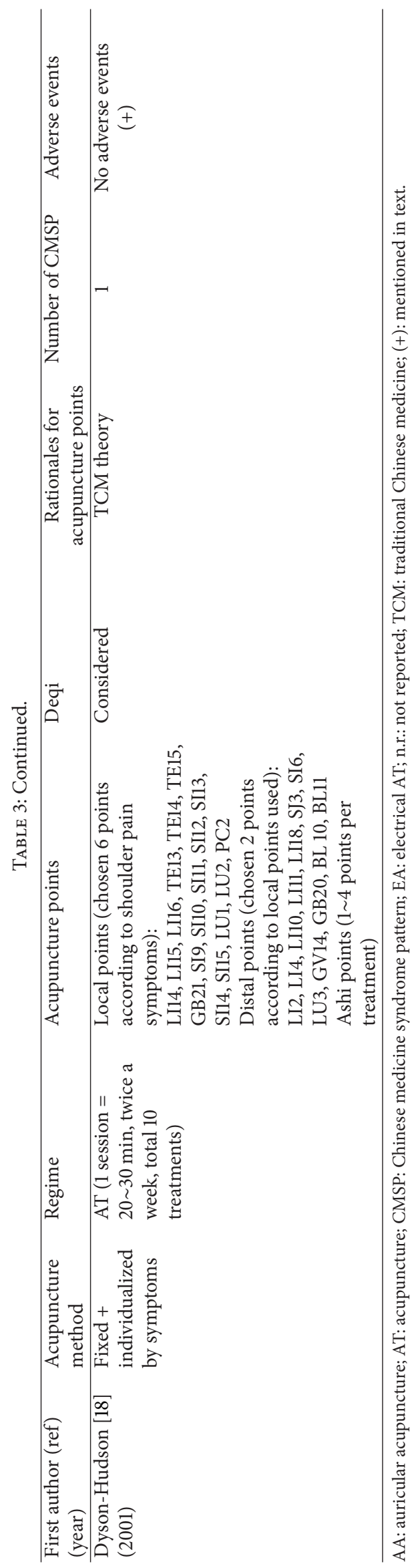




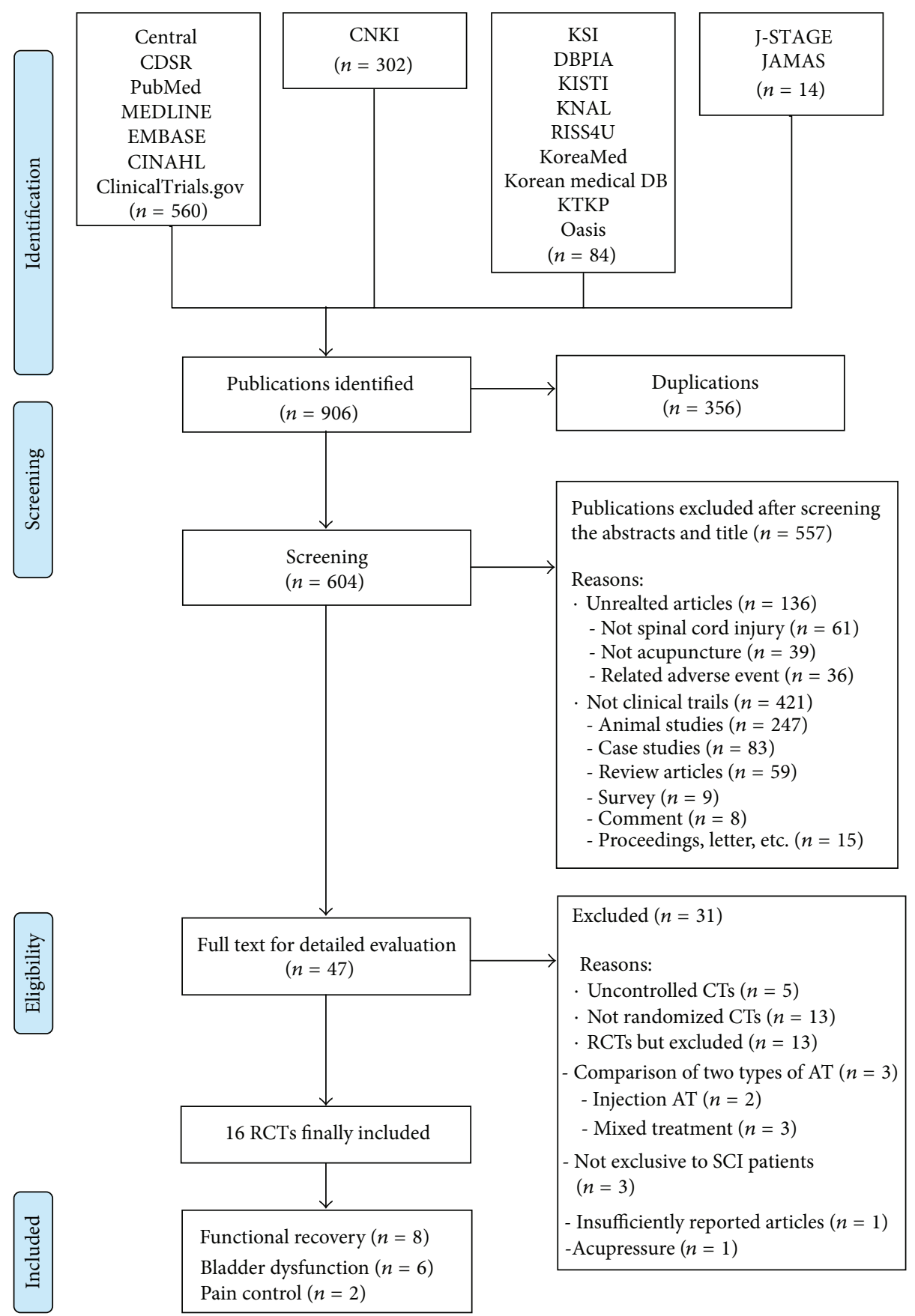

FIgURE 1: A flow chart describing the trial selection process. AT: acupuncture; CDSR: The Cochrane Database of Systematic Review; CENTRAL: The Cochrane Central Register of Controlled Trials; CNKI: China National Knowledge Infrastructure; CT: clinical trial; DB: database; KSI: Korean Studies Information; KISTI: Korea Institute of Science Technology Information; KNAL: Korean National Assembly Library; KTKP: Korean Traditional Knowledge Portal; RCT: randomized clinical trial; SCI: spinal cord injury. 
1. Functional recovery

1.1. ASIA score

1.1.1. Motor

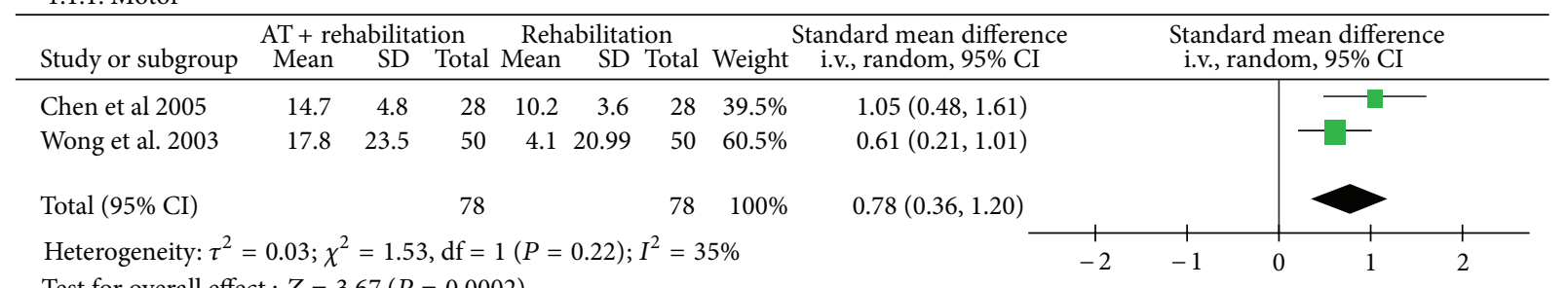

Test for overall effect : $Z=3.67(P=0.0002)$

Favours rehabilitation Favours AT + rehabilitation

1.1.2. Sensory

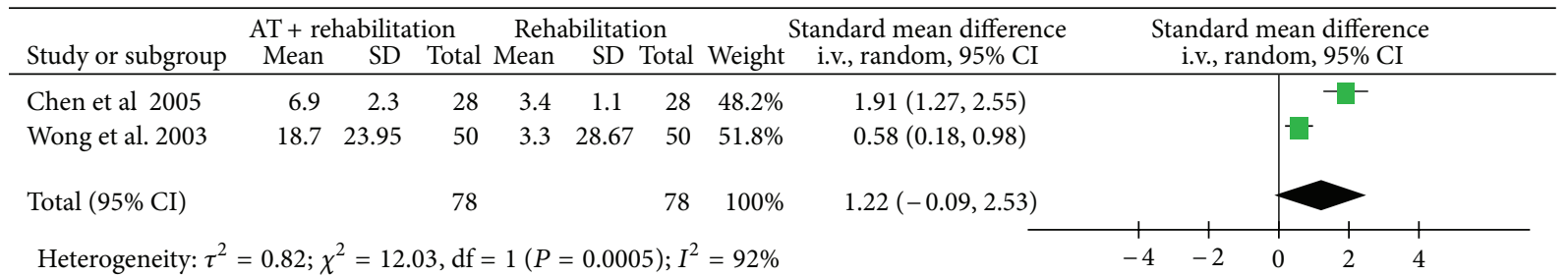

Test for overall effect : $Z=1.83(P=0.07)$

Favours rehabilitation Favours AT + rehabilitation

1.1.3. Pain

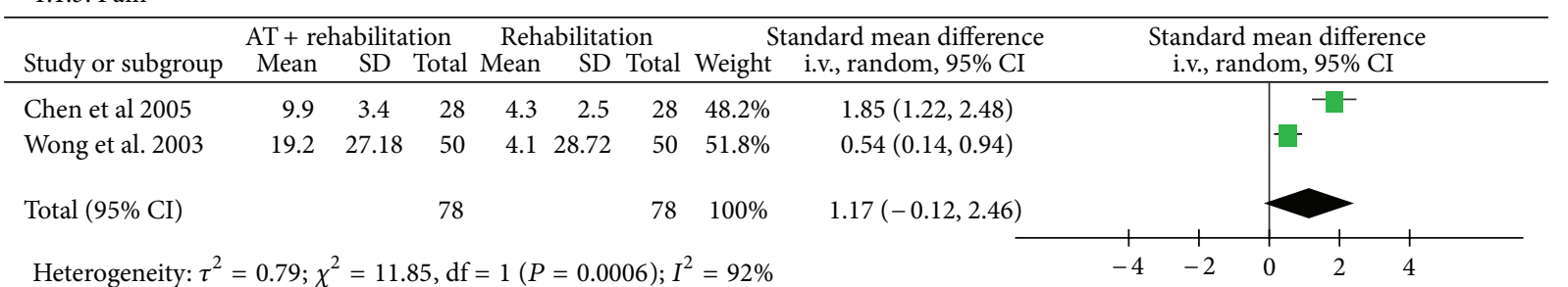

Test for overall effect : $Z=1.78(P=0.08)$

Favours rehabilitation Favours AT + rehabilitation

1.2. Total FIM score

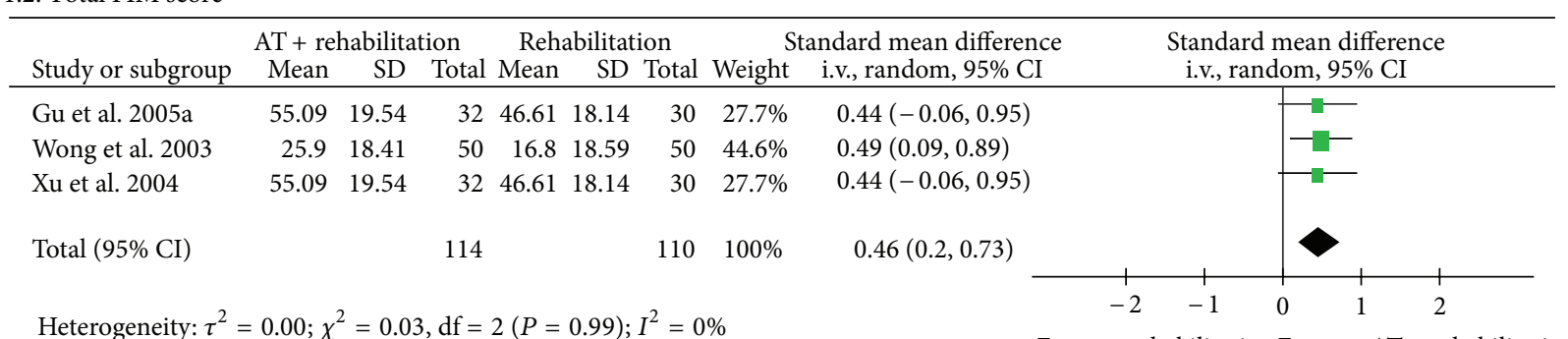

Test for overall effect : $Z=3.42(P=0.0006)$

Favours rehabilitation Favours AT + rehabilitation

2. Bladder dysfunction

2.1. Total efficacy rate

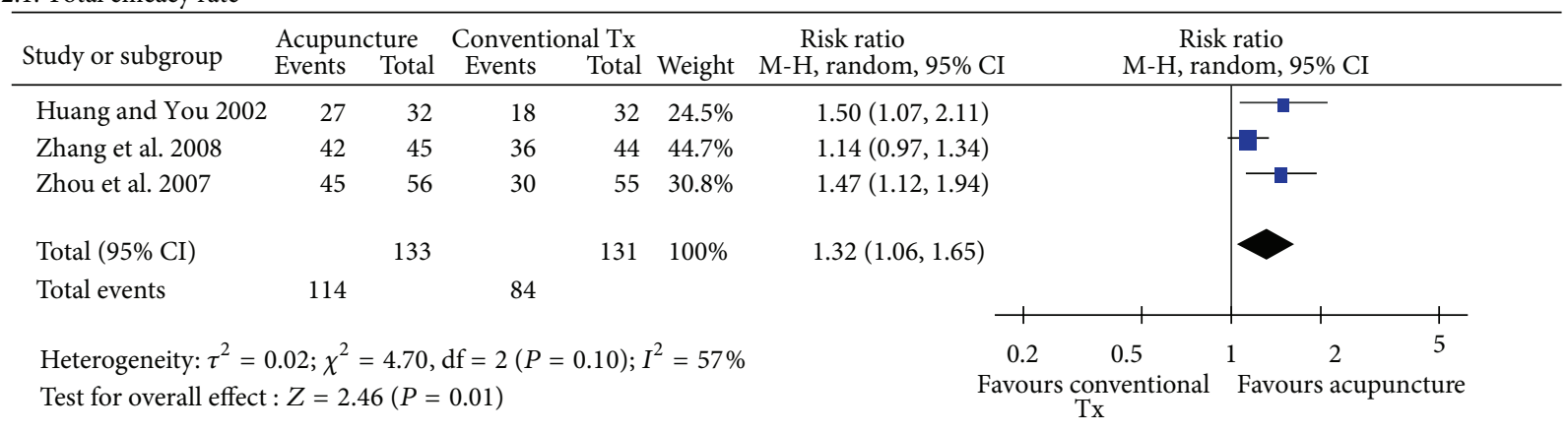

FIgURE 2: The meta-analysis of acupuncture for spinal cord injury and its complications. ASIA: American Spinal Injury Association; AT: acupuncture; FIM: functional independence measure; Tx: treatment. 
$\chi^{2}=1.53, P=0.22$, and $I^{2}=35 \%$; Figure 2) and total FIM scores (3 studies $[4,6,8]: n=224, \mathrm{SMD}=0.46,95 \% \mathrm{CI}$ of 0.20 to $0.73, P=0.0006$, heterogeneity: $\chi^{2}=0.03, P=0.99$, and $I^{2}=0 \%$; Figure 2).

\subsubsection{Bladder Dysfunction}

Acupuncture versus Conventional Treatment (3 RCTs). Three RCTs evaluated the effects of electrical acupuncture compared to conventional treatment (intramuscular neostigmine methylsulfate, intermittent catheterization, bladder training, etc.) $[11-13]$.

Two of the 3 RCTs used intramuscular neostigmine methylsulfate plus intermittent catheterization and bladder training $[11,12]$. One study showed that electrical acupuncture induced significantly greater improvement when compared to conventional treatment alone in terms of the total efficacy rate $(P=0.02$, Table 1$)$ [11]. However, the other study did not show a significant difference between treatment conditions $(P=0.11$, Table 1$)[12]$.

One of the 3 RCTs compared the effects of electrical acupuncture with conventional treatment (intermittent catheterization and bladder training) [13]. This study showed a significant positive effect for electrical acupuncture in terms of the total efficacy rate $(P=0.006$, Table 1$)$ and the levels of residual urine $(P<0.00001$, Table 1$)$ [13].

The meta-analysis of acupuncture versus conventional treatment showed a significant positive effect of acupuncture in terms of the total efficacy rate (3 studies [11-13]: $n=264$, $\mathrm{RR}=1.32,95 \% \mathrm{CI}$ of 1.06 to $1.65, P=0.01$, heterogeneity: $\chi^{2}=4.70, P=0.10$, and $I^{2}=57 \%$; Figure 2).

Acupuncture plus Conventional Treatment versus Conventional Treatment Alone (3 RCTs). Two RCTs compared the effects of electrical acupuncture plus intermittent catheterization and bladder training to intermittent catheterization and bladder training alone $[14,16]$. A recalculation of the mean difference $(\mathrm{MD})$ revealed that there was a significant positive effect for electrical acupuncture in terms of the total days needed to reach bladder balance $(P<0.05$, Table 1$)$ [14]; however, there was not a positive effect in bladder voiding function parameters (the frequency of urination, the maximum voided volume, bladder capacity, residual urine, and quality of life scores, Table 1) [16].

One RCT compared the effects of electrical acupuncture plus intermittent catheterization versus intermittent catheterization alone [15]. This study showed a positive effect in terms of the total efficacy rate $(P=0.007$, Table 1$)$ [15].

\subsubsection{Pain Control}

Acupuncture versus Sham Acupuncture (1 RCT). One RCT evaluated the effects of manual acupuncture with no manipulation at sites located at least 1 cun (1 Chinese anatomic inch, approximately $2.5 \mathrm{~cm}$ ) away from the established meridian and extra points [17]. The results showed that acupuncture did not significantly affect the PU-WUSPI and the NRS (Table 1) [17].
Acupuncture versus Trager Approach (1 RCT). One RCT assessed the effects of manual acupuncture compared to the Trager Approach (a form of bodywork and movement reeducation developed by Milton Trager) [18]. Our recalculation of the MD showed that there was no significant difference between the 2 treatments $(P>0.05$, Table 1$)[18]$.

3.4.4. The Safety Reporting of Acupuncture. Only three RCTs reported adverse events associated with acupuncture [7, 17, 18]. Two RCTs reported no adverse events [7, 18], and 1 trial reported minimal adverse effects without a detailed explanation [17].

\section{Discussion}

This is the first systematic review and meta-analysis of RCTs that fully evaluates the effectiveness of needle acupuncture for SCI and its complications. Of the 16 trials included in this paper, 8 trials studied the effects of acupuncture on functional recovery in SCI [3-10] and 8 trials that studied its effect on the secondary complications that follow SCI (6 trials for bladder dysfunction [11-16], and 2 trials for pain levels [17, 18]).

Based on the Cochrane risk of bias [33] and the PEDro scale [34], the methodological quality and design of the primary studies was mostly poor (only 2 of 16 were considered high quality, $12.5 \%)$. Of the 16 studies included in our review, 2 trials used appropriate sequence generation methods [17, 18]. Only one of the studies reported allocation concealment [9]. One RCT reported details of assessor- and patientblinding procedures [17]. Trials with inappropriate randomization were threatened by selection bias [50] and inadequate blinding tended to exaggerate the effects of treatment [33]. Researchers should conduct their trials according to the CONSORT statement [51]. In addition, 14 of the 16 RCTs originated from Chinese sources [3-16]. Several groups have shown that the majority of Chinese acupuncture studies report positive results $[52,53]$. Therefore, it is possible that a publication bias exists; although we searched extensively for all the studies that are relevant to this paper, we may have failed to conduct an analysis of publication bias [54]. This phenomenon casts a considerable doubt on the reliability of these data.

Ten trials compared acupuncture plus various conventional therapies to conventional therapies alone [4-10, 14-16], and 5 trials compared acupuncture to conventional therapies $[3,11-13,18]$. Such trial designs are open to bias because participants were not blinded. Sham-controlled trials could control nonspecific effects of acupuncture [55]. Only one trial adopted a penetrating sham control [17]. This shamcontrolled trial had a small sample size, and our recalculated power for this study was 0.326 . This finding indicates that the study lacked sufficient statistical power. Thus, these results limited our ability to evaluate the effectiveness of this treatment for various conditions in SCI. Additionally, nonpenetrating sham acupuncture may be more acceptable than penetrating sham $[56,57]$.

Although the effectiveness of acupuncture is unclear in SCI or its complications, acupuncture therapy is a relatively 
convenient and safe treatment for some conditions [58]. SCI patients who receive long-term rehabilitation or medication treatments may occasionally need a safe nonpharmacological treatment [59]. In this regard, acupuncture can be useful to treat SCI and its complications if patients experience side effects or have no (or a weak) response to a conventional treatment. Thus, we carefully present several points that must be discussed in future research.

First, although several animal studies of SCI have reported that acupuncture induces neuronal function recovery [60], an analgesic effect [61], and anti-inflammatory responses [62], the RCTs included in this paper might have used specific treatment conditions that may not have fully drawn the maximal benefit from acupuncture. To evaluate the effects of acupuncture on functional recovery in SCI, an appropriate set of treatment conditions which can maximize the therapeutic effect of acupuncture is highly recommended. Also, the conditions have to include the appropriate duration of treatment resulted from comparison of duration of the course of acupuncture treatment. Acupuncture as a (sole or adjunct) treatment for bladder dysfunction demonstrated a positive effect over all conventional treatments except one; while this is encouraging, this result is inconclusive due to the small sample size and low methodological quality. The sham trial for pain control that was reviewed failed to show any specific positive analgesic effect of acupuncture; however, this trial lacked statistical power. It is possible that acupuncture is an effective treatment for pain in SCI, as indicated by a previous rigorous review [31]. Thus, future high-quality trials on secondary conditions of SCI should be conducted to evaluate the potential effects of acupuncture [63].

Second, the prescription of acupuncture points was not consistent across studies (Table 3). Also, the degree of stimulation and the duration and frequency of acupuncture treatment were very various (Table 3 ). And we could not find the consistency of the Chinese medicine pattern in included trials. So it was difficult to estimate the correlation between the difference of acupuncture treatment and its therapeutic effectiveness. They might not have been optimal or might even be considered an underdosage of treatment for SCI [64]. As a matter of fact, the optimal prescription of acupuncture treatment (acupuncture point, degree of stimulation, frequency of treatment, and a number of treatment sessions) for SCI is a controversial issue amongst acupuncture experts. A standardized prescription of acupuncture for SCI or its complications is necessary.

Third, sham controls in acupuncture research for SCI may represent an ethical issue [65]. Therefore, an adjunctive treatment of acupuncture to conventional treatments for SCI can be evaluated by RCTs comparing acupuncture plus conventional treatment to sham acupuncture plus conventional treatment or through comparative effectiveness research (CER) [66].

\section{Conclusion}

The results of our systematic review and meta-analysis suggest that the evidence for the effectiveness of acupuncture as a symptomatic treatment for SCI and its complications is encouraging but limited. There is a great need to test the clinical implications of acupuncture for a number of SCI-related conditions. The efficacy of acupuncture for SCI and its complications must be studied using sham-controlled RCTs or CER with a standardized acupuncture procedure; such RCTs would conform to the recommendations of the CONSORT and STRICTA guidelines [51, 67].

\section{Conflict of Interests}

The authors report no financial or other relationships relevant to the subject of this paper.

\section{Acknowledgment}

This work was supported by a 2-year research Grant of The Pusan National University.

\section{References}

[1] National Center for Injury Prevention, Injury Fact Book, National Center for Injury Prevention and Control Centers for Disease Control and Prevention, Atlanta, GA, USA, 2006.

[2] L. Ottomanelli and L. Lind, "Review of critical factors related to employment after spinal cord injury: implications for research and vocational services," Journal of Spinal Cord Medicine, vol. 32, no. 5, pp. 503-531, 2009.

[3] Z. G. Chen, J. J. Zhang, Z. M. Wong, H. L. Li, and A. L. Zhang, "The clinical study of Governer vessel electroacupuncture treatment on the lower extremity spasticity in patients with spinal cord injury," Journal of Clinical Acupuncture and Moxibustion, vol. 11, no. 6, pp. 6-7, 1995.

[4] A. M. K. Wong, C. P. Leong, T. Y. Su, S. W. Yu, W. C. Tsai, and C. P. C. Chen, "Clinical trial of acupuncture for patients with spinal cord injuries," American Journal of Physical Medicine and Rehabilitation, vol. 82, no. 1, pp. 21-27, 2003.

[5] X. M. Cui, X. M. Pan, X. M. Zhang, H. Z. Deng, and S. Y. Yao, "The rehabilitation function of electroacupuncture treatment in patients with traumatic spinal cord injury," Journal of Guangdong Medical College, vol. 22, no. 2, pp. 170-171, 2004.

[6] S. Xu, X. Gu, and M. Gu, "Study on effects of electrical acupuncture. Stimulation on activities of daily living in patients with spinal cord injury," Journal of Traditional Chinese Medicine, no. 1, p. 30, 2004.

[7] L. X. Chen, J. F. Duan, X. R. Zhan, Q. Xie, and Q. H. Long, "Electrical and auricular acupucture for patients with acute spinal cord injury," Clinical Journal of Rehabilitation Theory and Practice, vol. 11, no. 11, pp. 934-935, 2005.

[8] X. D. Gu, Y. H. Yao, M. Gu, J. M. Fu, Y. Ren, and H. K. Yin, "Clinical study on functional independence measure for patients with spinal cord injury of trauma treated by electroacupuncture," Acupuncture Research, vol. 30, no. 3, pp. 175$178,2005$.

[9] C. H. Ma, "The effects of combination treatment of electroacupuncture and weight lift walking exercise on the walking function of spinal cord injury patients," Chinese Journal of Clinical Rehabilitation, vol. 9, no. 17, p. 186, 2005.

[10] G. Sheng, J. Huo, and L. Wang, "Clinical observation on electroacupuncture treatment in 24 cases of spinal cord injury," Chinese 
Journal of Traditional Medical Science and Technology, vol. 16, no. 2, p. 130, 2009.

[11] Z. G. Huang and B. You, "Acupuncture treatment on 32 cases of urinary retention after spinal cord injury," Shanghai Journal of Acupuncture and Moxibustion, vol. 21, no. 2, p. 31, 2002.

[12] S. Zhang, "Clinical observation on electro-acupuncture treatment in 45 cases of neurogenic bladder following spinal cord injury," Zhejiang Journal of Traditional Chinese Medicine, vol. 43, no. 11, p. 654, 2008.

[13] L.-Y. Zhou, Z.-G. Yu, J Li et al., "Therapeutic effects of electroacupuncture treatment on posterior sacral foramia and sacral hiatus on neurogenic voiding dysfunction," Journal of Harbin Medical University, vol. 41, no. 4, pp. 367-369, 2007.

[14] P. T. Cheng, M. K. Wong, and P. L. Chang, "A therapeutic trial of acupuncture in neurogenic bladder of spinal cord injured patients-a preliminary report," Spinal Cord, vol. 36, no. 7, pp. 476-480, 1998.

[15] X. D. Gu, J. M. Fu, and Y. H. Yao, "Clinical observation of electroacupuncture on eight-liao points in 64 bladder dysfunction patients with spinal cord injury," Journal of Clinical Acupuncture and Moxibustion, vol. 21, no. 4, pp. 47-48, 2005.

[16] Y. Liu, M. Xie, W. Feng, X. Zhou, and L. Zhou, "Case study on the effect of Hua-Tuo-Jia-Ji-Xue acupuncture treatment of patient with bladder dysfunction after spinal cord injury," Shandong Medical Journal, vol. 49, no. 12, p. 95, 2009.

[17] T. A. Dyson-Hudson, P. Kadar, M. LaFountaine et al., "Acupuncture for chronic shoulder pain in persons with spinal cord injury: a small-scale clinical trial," Archives of Physical Medicine and Rehabilitation, vol. 88, no. 10, pp. 1276-1283, 2007.

[18] T. A. Dyson-Hudson, S. C. Shiflett, S. C. Kirshblum, J. E. Bowen, and E. L. Druin, "Acupuncture and trager psychophysical integration in the treatment of wheelchair user's shoulder pain in individuals with spinal cord injury," Archives of Physical Medicine and Rehabilitation, vol. 82, no. 8, pp. 1038-1046, 2001.

[19] R. P. Yezierski, "Spinal cord injury pain: spinal and supraspinal mechanisms," Journal of Rehabilitation Research and Development, vol. 46, no. 1, pp. 95-108, 2009.

[20] B. S. Bregman, J. V. Coumans, H. N. Dai et al., "Transplants and neurotrophic factors increase regeneration and recovery of function after spinal cord injury," Progress in Brain Research, vol. 137, pp. 257-273, 2002.

[21] N. Attal, G. Mazaltarine, B. Perrouin-Verbe, and T. Albert, "Chronic neuropathic pain management in spinal cord injury patients. What is the efficacy of pharmacological treatments with a general mode of administration? (oral, transdermal, intravenous)," Annals of Physical and Rehabilitation Medicine, vol. 52, no. 2, pp. 124-141, 2009.

[22] C. Norrbrink Budh, J. Kowalski, and T. Lundeberg, "A comprehensive pain management programme comprising educational, cognitive and behavioural interventions for neuropathic pain following spinal cord injury," Journal of Rehabilitation Medicine, vol. 38, no. 3, pp. 172-180, 2006.

[23] S. R. Silverman, L. A. Schertz, H. K. Yuen, J. D. Lowman, and C. S. Bickel, "Systematic review of the methodological quality and outcome measures utilized in exercise interventions for adults with spinal cord injury," Spinal Cord, vol. 10, no. 50, pp. 718-727, 2012.

[24] T. Huston, J. Gassaway, C. Wilson, S. Gordons, J. Koval, and A. Schwebe, "The SCIRehab project: treatment time spent in SCI rehabilitation. Psychology treatment time during inpatient spinal cord injury rehabilitation," Journal of Spinal Cord Medicine, vol. 34, no. 2, pp. 196-204, 2011.
[25] N. B. Finnerup and T. S. Jensen, "Spinal cord injury painmechanisms and treatment," European Journal of Neurology, vol. 11, no. 2, pp. 73-82, 2004.

[26] P. T. Dorsher and P. M. McIntosh, "Acupuncture's effects in treating the sequelae of acute and chronic spinal cord injuries: a review of allopathic and traditional Chinese medicine literature," Evidence-based Complementary and Alternative Medicine, vol. 2011, Article ID 428108, 2011.

[27] N. Robinson, A. Lorenc, W. Ding, J. Jia, M. Bovey, and X. M. Wang, "Exploring practice characteristics and research priorities of practitioners of traditional acupuncture in China and the EU-A survey," Journal of Ethnopharmacology, vol. 140, no. 3, pp. 604-613, 2012.

[28] V. Wong, D. K. Cheuk, S. Lee, and V. Chu, "Acupuncture for acute management and rehabilitation of traumatic brain injury," Cochrane Database of Systematic Reviews, vol. 5, article CD007700, 2011.

[29] X. F. Zhao, Y. Du, P. G. Liu, and S. Wang, "Acupuncture for stroke: evidence of effectiveness, safety, and cost from systematic reviews," Topics in Stroke Rehabilitation, vol. 19, no. 3, pp. 226-233, 2012.

[30] B. C. Shin, M. S. Lee, J. C. Kong, I. Jang, and J. J. Park, "Acupuncture for spinal cord injury survivors in Chinese literature: a systematic review," Complementary Therapies in Medicine, vol. 17, no. 5-6, pp. 316-327, 2009.

[31] D. D. Cardenas and E. R. Felix, "Pain after spinal cord injury: a review of classification, treatment approaches, and treatment assessment," PM and R, vol. 1, no. 12, pp. 1077-1090, 2009.

[32] D. Moher, A. Liberati, J. Tetzlaff, and D. G. Altman, "Preferred reporting items for systematic reviews and meta-analyses: the PRISMA statement," International Journal of Surgery, vol. 8, no. 5, pp. 336-341, 2010.

[33] J. Higgins and S. Green, "Analysing and presenting results," in Cochrane Handbook for Systematic Reviews of Interventions 4.2.6 [updated September 2006], pp. 79-165, John Wiley \& Sons, Chichester, UK, 2008.

[34] C. G. Maher, C. Sherrington, R. D. Herbert, A. M. Moseley, and M. Elkins, "Reliability of the PEDro scale for rating quality of randomized controlled trials," Physical Therapy, vol. 83, no. 8, pp. 713-721, 2003.

[35] N. C. Foley, S. K. Bhogal, R. W. Teasell, Y. Bureau, and M. R. Speechley, "Estimates of quality and reliability with the physiotherapy evidence-based database scale to assess the methodology of randomized controlled trials of pharmacological and nonpharmacological interventions," Physical Therapy, vol. 86, no. 6, pp. 817-824, 2006.

[36] M. Paci, G. Matulli, M. Baccini, L. A. Rinaldi, and S. Baldassi, "Reported quality of randomized controlled trials in neglect rehabilitation," Neurological Sciences, vol. 31, no. 2, pp. 159-163, 2010.

[37] Y. Zhang, "Relationship between acupuncture and recovery of spinal cord injury," Journal of Traditional Chinese Orthopedics and Traumatology, vol. 10, no. 6, pp. 58-59, 2002.

[38] L. Y. Zhou, J. Li, C. M. Li et al., "Observation on therapeutic effect of electroacupuncture at points Baliao and Huiyang (BL 35 ) on retention of urine induced by spinal cord injury," Chin Acupunct Moxibustion, vol. 26, no. 4, pp. 237-239, 2006.

[39] W. W. Chen and N. Li, "Acupuncture for the lower extremity spasm induced by spinal injury: random controlled trial of single case," Chinese Acupuncture and Moxibustion, vol. 30, no. 6, pp. 473-477, 2010. 
[40] X. F. Liu, Z. A. Liao, W. H. Deng, X. L. Guan, and J. Luo, "Effects of drug injection at eight-liao point combined with bladder function training on bladder dysfunction due to spinal cord injury," Chinese Journal of Clinical Rehabilitation, vol. 9, no. 25, pp. 142-143, 2005.

[41] S. T. Xing, D. Wang, X. H. Wen et al., "Clinical research of electroacupuncture combined with acupoint-injection of botulinum toxin A in treating the muscle spasticity by spinal cord injury," China Journal of Orthopaedics and Traumatology, vol. 23, no. 5, pp. 350-353, 2010.

[42] L. M. Shan, X. J. Wu, and C. M. Yang, "Clinical effects of acupuncture combined with Tuina manipulation for the treatment of paralysis induced by traumatic spinal cord injury," Zhong Yi Zheng Gu, vol. 11, no. 9, pp. 14-15, 1999.

[43] C. Yan, "The effect of antispasmodic on the physical functions of cases with spinal paraplegia," Modern Rehabilitation, vol. 3, no. 10, pp. 1162-1163, 1999.

[44] G. H. Zhang and S. F. Sun, "Clinical effects of Yi-qi-huo-Xuetang plus acupuncture treatment on the 20 patients with spinal cord injury," Hebei Chinese Medicine, vol. 26, no. 1, pp. 21-22, 2004.

[45] X. Y. Teng, "Clinical effects of acupuncture treatment on the bladder dysfunction," Journal of Clinical Acupuncture and Moxibustion, vol. 19, no. 4, p. 17, 2003.

[46] Z. H. Yue, W. L. Liu, and J. M. Jiang, "Acupuncture treatment and functional assessment on the spasticity of extremity," Chinese Journal of Rehabilitation Medicine, vol. 17, no. 3, pp. 177178, 2002.

[47] W. Zhang, W. L. Liu, and Y. L. Zhao, "Clinical study of 52 cases of spasmodic paralysis," Zhong Guo Yi Kan, vol. 34, no. 3, pp. 52-53, 1999.

[48] Z. Liao, "Comparison analysis of cervical disease treatment by acupuncture and maneuver therapy," Chinese Journal of Clinical Rehabilitation, vol. 7, no. 6, p. 1049, 2003.

[49] C. W. Liu, C. H. Chen, Y. F. Huang, and M. H. Huang, "Auricular acupressure as a treatment for neuropathic pain in patients with spinal cord injury," European Journal of Pain Supplements, vol. 4, no. 1, p. 132, 2010.

[50] P. Jüni, D. G. Altman, and M. Egger, "Systematic reviews in health care: assessing the quality of controlled clinical trials," British Medical Journal, vol. 323, no. 7303, pp. 42-46, 2001.

[51] D. Moher, S. Hopewell, K. F. Schulz et al., "CONSORT 2010 Explanation and Elaboration: updated guidelines for reporting parallel group randomised trials," Journal of Clinical Epidemiology, vol. 63, no. 8, pp. e1-e37, 2010.

[52] A. Vickers, N. Goyal, R. Harland, and R. Rees, "Do certain countries produce only positive results? A systematic review of controlled trials," Controlled Clinical Trials, vol. 19, no. 2, pp. 159-166, 1998.

[53] J. L. Tang, S. Y. Zhan, and E. Ernst, "Review of randomised controlled trials of traditional Chinese medicine," British Medical Journal, vol. 318, no. 7203, pp. 160-161, 1999.

[54] R. Joober, N. Schmitz, L. Annable, and P. Boksa, "Publication bias: what are the challenges and can they be overcome?" Journal of Psychiatry \& Neuroscience, vol. 37, no. 3, pp. 149-152, 2012.

[55] E. A. Tough, A. R. White, S. H. Richards, B. Lord, and J. L. Campbell, "Developing and validating a sham acupuncture needle," Acupuncture in Medicine, vol. 27, no. 3, pp. 118-122, 2009.
[56] I. Lund and T. Lundeberg, "Are minimal, superficial or sham acupuncture procedures acceptable as inert placebo controls?" Acupuncture in Medicine, vol. 24, no. 1, pp. 13-15, 2006.

[57] I. Lund, J. Näslund, and T. Lundeberg, "Minimal acupuncture is not a valid placebo control in randomised controlled trials of acupuncture: a physiologist's perspective," Chinese Medicine, vol. 4, article 1, 2009.

[58] A. D. Furlan, F. Yazdi, A. Tsertsvadze et al., "A systematic review and meta-analysis of efficacy, cost-effectiveness, and safety of selected complementary and alternative medicine for neck and low-back pain," Evidence-Based Complementary and Alternative Medicine, vol. 2012, Article ID 953139, 61 pages, 2012.

[59] M. Heutink, M. W. M. Post, M. M. Wollaars, and F. W. A. Van Asbeck, "Chronic spinal cord injury pain: pharmacological and non-pharmacological treatments and treatment effectiveness," Disability and Rehabilitation, vol. 33, no. 5, pp. 433-440, 2011.

[60] Y. C. Chen, W. L. Qi, and K. M. Kong, "Effect of electroacupunture on expression changes of nerve growth factor and receptor in rats with acute spinal cord injury," Chinese Journal of Clinical Rehabilitation, vol. 10, no. 11, pp. 129-131, 2006.

[61] H. Y. Kim, J. Wang, I. Lee, H. K. Kim, K. Chung, and J. M. Chung, "Electroacupuncture suppresses capsaicin-induced secondary hyperalgesia through an endogenous spinal opioid mechanism," Pain, vol. 145, no. 3, pp. 332-340, 2009.

[62] D. C. Choi, J. Y. Lee, Y. J. Moon, S. W. Kim, T. H. Oh, and T. Y. Yune, "Acupuncture-mediated inhibition of inflammation facilitates significant functional recovery after spinal cord injury," Neurobiology of Disease, vol. 39, no. 3, pp. 272-282, 2010.

[63] E. Ernst, M. H. Pittler, B. Wider, and K. Boddy, "Acupuncture: its evidence-base is changing," American Journal of Chinese Medicine, vol. 35, no. 1, pp. 21-25, 2007.

[64] A. White, M. Cummings, P. Barlas et al., "Defining an adequate dose of acupuncture using a neurophysiological approach-a narrative review of the literature," Acupuncture in Medicine, vol. 26, no. 2, pp. 111-120, 2008.

[65] S. D. Pearson, "Placebo-controlled trials, ethics, and the goals of comparative effectiveness research: comment on 'lack of head-to-head trials and fair control arms"', Archives of Internal Medicine, vol. 172, no. 3, pp. 244-245, 2012.

[66] C. M. Witt, E. Manheimer, R. Hammerschlag et al., "How well do randomized trials inform decision making: systematic review using comparative effectiveness research measures on acupuncture for back pain," PLoS One, vol. 7, no. 2, article e32399, 2012.

[67] H. MacPherson, D. G. Altman, R. Hammerschlag et al., "Revised STandards for Reporting Interventions in Clinical Trials of Acupuncture (STRICTA): Extending the CONSORT statement," Journal of Evidence-Based Medicine, vol. 3, no. 3, pp. 140-155, 2010. 


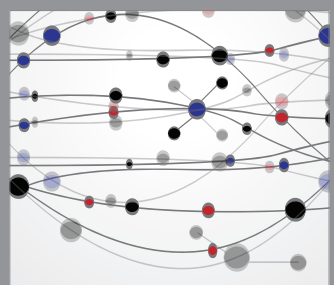

The Scientific World Journal
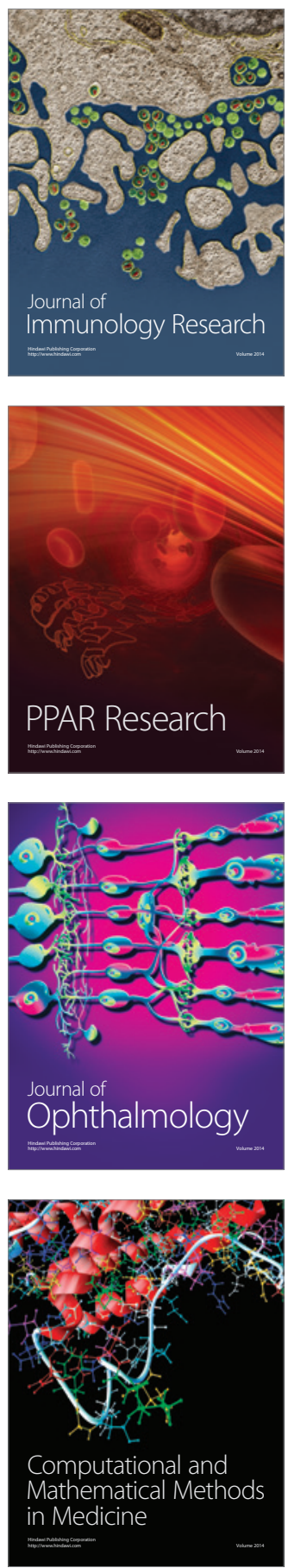

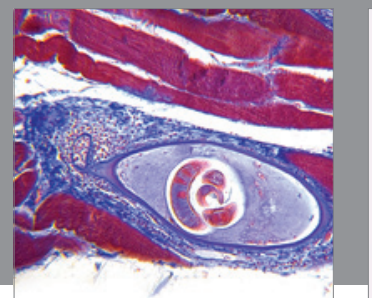

Gastroenterology

Research and Practice
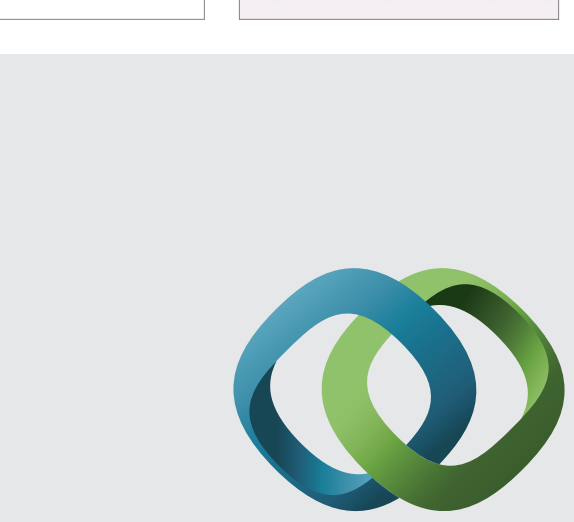

\section{Hindawi}

Submit your manuscripts at

http://www.hindawi.com
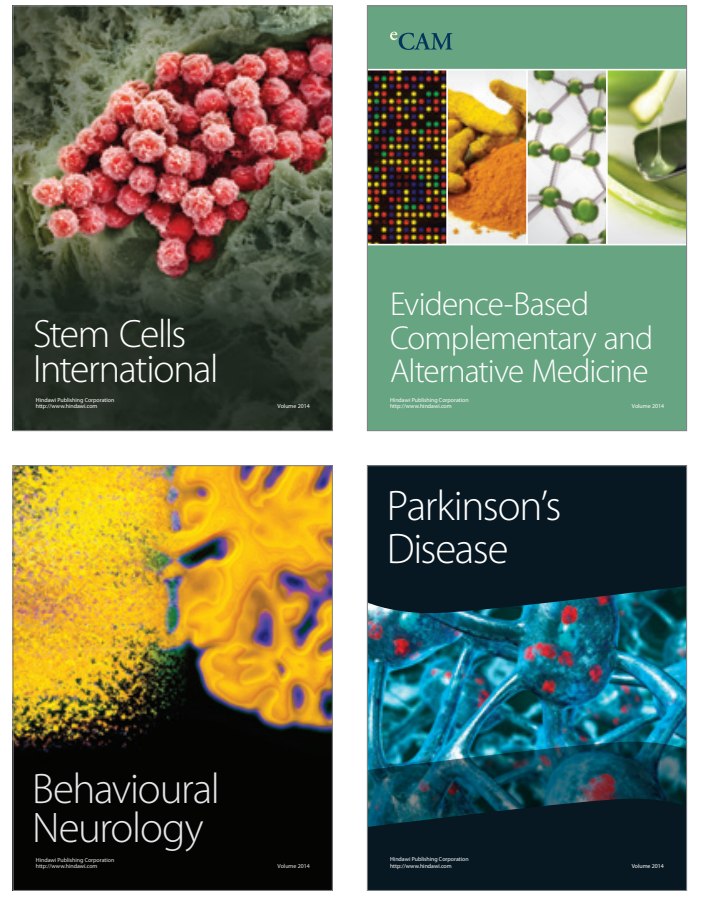
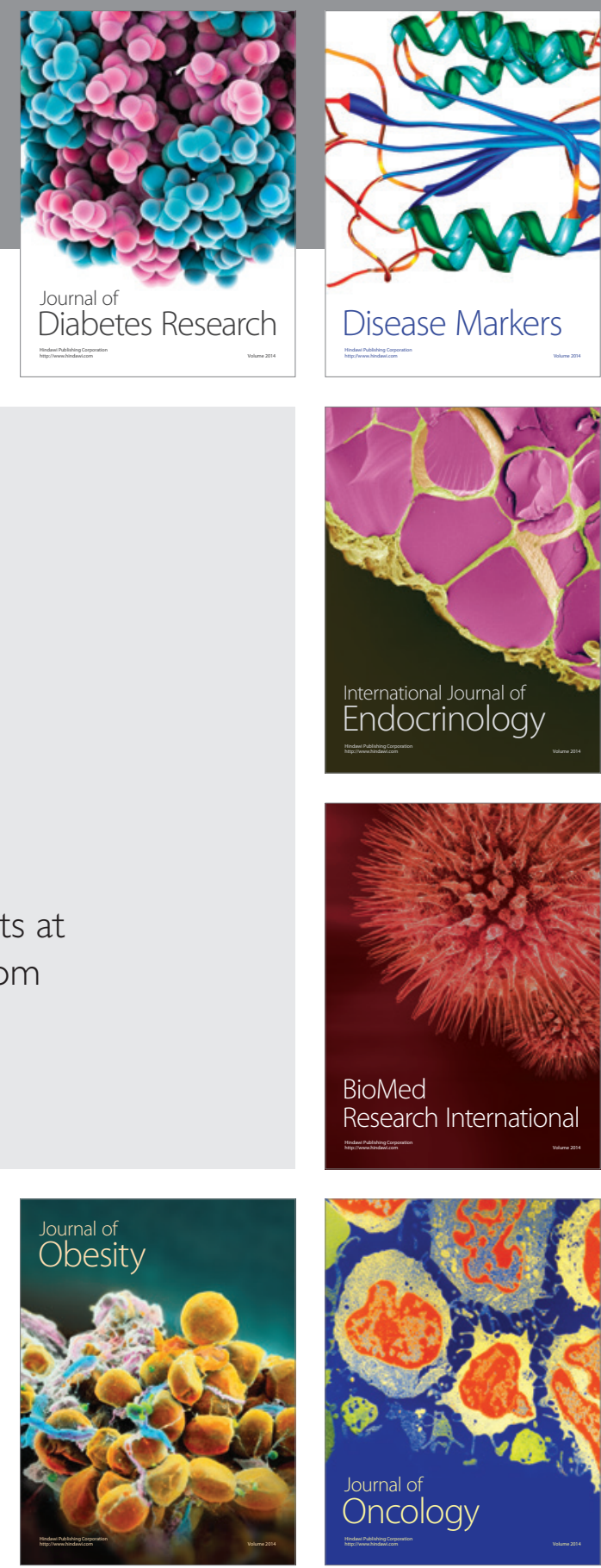

Disease Markers
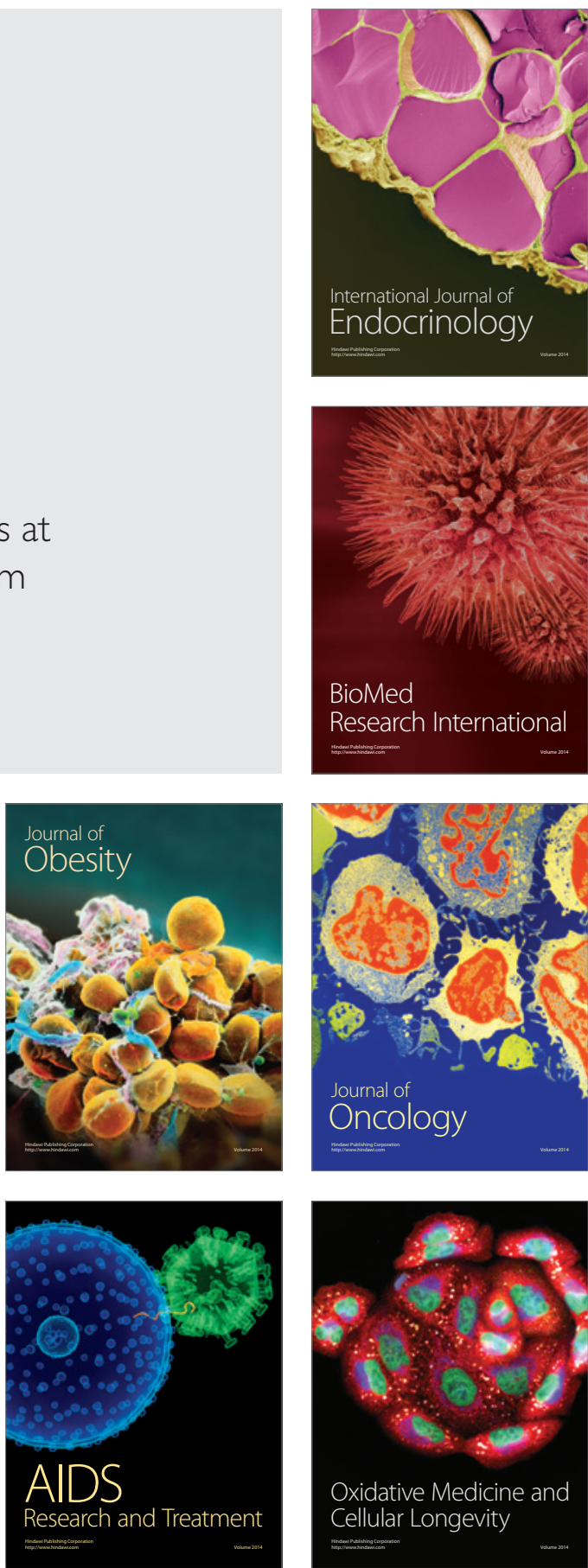